\title{
Comparing Gain and Loss Framed Message Texting (SMS) on Foot Self- Care Behaviors Among Women with Type 2 Diabetes
}

\author{
Z Baji ${ }^{1,}{ }^{*} ;$ F Zamani Alavijeh ${ }^{2}$; Gh Shakerinejad ${ }^{3}$; M Tehrani $^{4}$ \\ 'MSc of Health Education, Health Education Research Department, ACECR-Khuzestan, Ahvaz, Iran \\ ${ }^{2}$ Assistant Professor of Health Education, Department of Public Health, Social Determinants of Health Research Centre, School of Health, Ahvaz Jundishapur Medical Sciences \\ University, Ahvaz, Iran \\ ${ }^{3}$ Associate Professor of Health Education, Health Education Research Department, ACECR-Khuzestan, Ahvaz, Iran \\ ${ }^{4}$ MSc of Nutrition, Health Education Research Department, ACECR-Khuzestan, Ahvaz, Iran \\ * Corresponding author: Z Baji, MSc of Health Education, Health Education Research Group, ACECR-Khuzestan, Ahvaz, Iran. P. O. Box: 6135733773, Tel: +98-6133330023, \\ E-mail: zahrabaji65@yahoo.com
}

Received: 11 Dec 2016

Accepted: 01 Jan 2017

Epub: 23 Feb 2017

Ppub: 15 Jan 2018

\begin{abstract}
Background: The effectiveness of the educational message for motivating to change behavior may be greater than the actual content of a message, depending on how the message is designed.

objectives: The purpose of this study was to compare the effect of gain and loss messages via SMS mobile phone on foot self-care behaviors in women with Type 2 diabetes who were referred to the Ahwaz diabetic clinic.

Methods: In this randomized controlled trial study, 189 Type 2 diabetes patients were selected by convenience sampling and then randomly divided into 3 exp. groups and cont. group. Two experimental groups received gain and loss educational message of foot care behaviors via mobile phone short message service for 2 months. Research data were collected through interviews using demographic and disease characteristics questionnaires and the summary of diabetes self-care activities were measured. The collected data were analyzed by using the SPSS 16 software, paired t-test, one-way ANOVA, and Chi-square at the level of 0.05 significance.

Results: Before the intervention disease characteristics, individual variables and the average score of foot care behaviors of the study groups were similar. After the intervention, a significant increase occurred in the mean score foot care in experimental groups $(\mathrm{P}=0.001)$. It was also shown that this increase in loss framed message group is significantly more than the gain message $\operatorname{group}(\mathrm{P}=0.01)$.

Conclusions: According to the results, designing and implementing educational programs based on mobile phone short message service and loss framed messages could improve foot-care behaviors in diabetic patients.
\end{abstract}

Keywords: Type 2 Diabetes; Framing Message; Short Message Service; Women; Gain and Loss Framed Message 